\title{
THE EFFECT OF RADIATION ON THE LONG TERM PRODUCTIVITY OF A PLANT BASED CELSS.
}

\author{
B.G. Thompson and B.II. Lake \\ Biotechnology Department, Alberta Research Council, Edmonton, Alberta, Canada
}

\begin{abstract}
Mutations occur at a higher rate in space than under terrestrial conditions, primarily due to an increase in radiation levels. These nutations may effect the productivity of plants found in a controlled ecological hife support system (CEISS). Computer simulations of plants with different ploidies, modes of reproduction, lethality thresholds, viability thresholds and susceptibilitics to radiation induced mutations were performed under space normal and solar fare conditions. These simulations identified plant characteristics that would enable plants to retain high productivities over time in a CELSS.
\end{abstract}

\section{INTRODUCTION}

Controlled ecological life support systems (CELSS) will form at least part of the overall life support system used for deep space missions /1/. These missions will have a duration of years, a limited crew, and probably severe mass limitations. CELSS designed to operate in space must perform within these constraints as well as those imposed by the space environment.

Plants will probably be used to perform at least some of the functions in a CELSS, including biomass generation, carbon dioxide reduction, oxygen regeneration, and waste recycling / 1 . The selection of plant species for use in a CELSS will take into consideration several factors. To minimize CELSS energy, size and mass requirements, the net productivity of the plants to be grown should be maximized with respect to these parameters. The biomass obtained from the plant should meet as many human metabolic requirements as possible. The plant should be capable of being cultivated ivith little luman intervention and should require only a simple growth and harvesting system. The plant must suffer little or no productivity losses due to nultiple generation growth in the space environment.

The environment found in space will effect the growth of plants. Microgravity can induce gravitropic effects during the development of the plant /2/. Radiation can cause phenotypic and/or genotypic effects in plants /3-7/. Any CELSS deployed in space will not have large excess capacities for production built into it, as a result of mass and volume restrictions. If any aspect of the space cnviromnent reduces the production levels of a CELSS, unscheduled terminations of fight personnel may occur.

'This study examines the genotypic effect of radiation on the productivity of a plant based CELSS at space normal levels as well as during solar flare activity.

\section{COMPUTER MODEL DEFINITION}

Plants exhibit large variations in structural characteristics, gene numbers, reproductive unit type and weight and other attributes. These characteristic variations were standardized in order to compare the eflect of radiation on plants that vary in reproduclive mode, ploidy, viability thresholds, lethality thresholds, and susceptibility to mutation at constant radiation levels. Therefore any plant used in the model may not truly represent any one real plant species.

\section{Multi-generation Operations}

The model examined the nulti-generation effect of radiation induced mutations on the productivity of an isolated, size limited CELSS containing one species of plant. In each generation, a maximum of 100 plants were grown. $\Lambda$ limited plant number was selected to ensure that both general trends in system productivity and the skewing effect of random events could be detected. Each plant could produce an upper bound of 10 reproductive units (RUs). This number represents a compromise between the usually smaller number of RUs produced by vegetative plants and the usually larger number of RUs produced by seed producing plants. A subset of the RUs produced by these plants were used to start the next generation of plants, with the remainder being used as a food product. Key variables that affect the food product yield were recorded for cach plant generation. For each generation these included : the number of plants grown ( $\max 100)$, the total number of RUs produced (max 1000), net system productivity (max 900 ), number of RUs that were germinated (max 100), the number of RUs that. failed to germinate, the number of germinated RUs that reached RU production, and the number of gene mutations that occurred.

A majority of the model variables were assigned static values for the entirety of a multi-generation run. Configuralion choices included the modelled plant's (MPs) ploidy, reproductive mode, reproductive selection criterion, lethality threshold, viability threshold, genes subject to mutations, and frequency of gene mutation outcomes. The model assumed that productivity, viability, and lethality were under genetic control only and were not subject to phenotypic variations. Mutation frequency could be altered between generations to reflect solar fare conditions, although a constant mutation rate would be typical for most situations. The plants used for generation zero normally contained fully functional (wild type) genes, although specific genetic deficiencies could be induced before beginning a multi-generation run. 


\section{Modelled Plant (MP) Characteristics}

MPs contained genes that express only three traits considered essential for food production. Productivity measured total food product produced over time by plants that reach productive status. Genes that reduce RU weights, RU number, or bionass weight and increase growth periods were considered to reduce productivity. Productivity was measured as the RU number, assuming constant RU weight, with a MP producing a maximum of 10 RUs. Viability measured the proportion of RUs produced that were capable of germinating. Genes affecting viability reduced germination frequencies. Lethality measured the proportion of plants after germination that would die prior to reaching adulthood due to gene dysfunction. Genes affecting lethality were considered to be those that kill the plant or simply prevent it from producing food.

A MP contained 60 genes per chromosome, with the three MP genetic traits being represented by 20 genes per chromosome each. These 20 genes were divided into two gene clusters, each composed of four different sized gene systems. Gene systems consisted of one, two, three or four genes, which yields a total of 10 genes per gene cluster. This gene number was selected to ensure that gene duplication effects would occur and that differences between various plant types could be detected. The ploidy of MPs could be varied to be diploid, tetraploid or hexaploid.

MPs could reproduce in one of four diferent modes; vegetatively, by anther culture, by cross fertilization, or by self fertilization. Some modes are easier to implement than others : anther culture represents a high technology, labor intensive means of reproduction, while cross fertilization may require mechanical assistance due to a lack of insect or wind mediated cross pollination. During sexual reproduction (cross or self fertilization) or anther culture where ganetes were required for reproduction, gametes were produced by meiosis with all genes segregating independently. In anther culture reproduction, one gamete was produced for the plant and the RU was produced from two copies of this gamete. In cross fertilization reproduction, the plant chosen to reproduce was paired with another plant that. was randonly or selectively choscu. A gamete from each plant was combined to produce the new RU. In self fertilization reproduction, two gametes were prodiced from the same plant, and combined to form the new RU. In vegetative reproduction, RUs produced were clones of the parent plant.

Mutations

Genes in the MPs had four different states. Wild type genes were $100 \%$ functional. A leaky mutant gene was $50 \%$ functional, but would not impair the performance of other genes in its gene set. A recessive mutant gene was $0 \%$ functional, and would also not impair the performance of other genes in its gene set. A recessive dominant mutant gene had its function altered to the extent that it completely inhibited the function of all other genes for this trait. Positive mutations that altered genes so they performed better than wild type genes were not considered.

The software simulated various plant environments througla three separate gene mutation control switches. Immunity to mutations was achieved for any gene in a cliromosome via a simple mutation on/off switch. This allowed mutations to occur only in a single trait if desired. Mutation frequency was variable for each generation of plants. When a mutation occurred, the type of gene transformation that occurred could be varied in frequency for each of the four states (wild type, leaky, recessive, and recessive dominant.)

\section{RESULTS AND DISCUSSION}

The plants considered for use in a CELSS are in many cases available in different ploidies or may reproduce by a variety of reproductive modes. Genotypes within a species may also exhibit different levels of mutations under equal radiation flux conditions. In these cases, and in cases where different plant species may be used in a CELSS, computer simulations can aid the selection of plant types that will tend to suffer the least losses in net system productivity due to radiation induced mutations. For all simulation runs described here, the mutation rates were increased until differences, if any, between differing plant systems were noled.

\section{Nonflare Conditions}

In nonflare conditions the radiation flux encountered in space can be expected to be relatively constant. Space missions that are performed in periods of low solar activity can therefore expect relatively constant mutation rates to occur in each cultivar of the plant species used in a CELSS.

\section{The effect of variation in mutation frequency on net systen productivity in nonflare conditions.}

The mutation frequency that a particular genotype will experience under nonflare conditions is importaut in determining whetler or not it should be selected as a potential plant in the CELSS used. For all types of reproduction, regardless of ploidy, increasing the mutition frequency experienced by the plant had a negative effect over time on net system productivities (Figure 1).

The effect of ploidy on net system productivity in nonflare conditions.

Sonc plant genera, for example Triticum (wheat), contain species that have different ploidies. Lines with different ploidies can also be generated by tissue culture techniques. Under conditions of high system mutation frequencies, increasing ploidy for plants reproducing by any type of reproduction resulted in ligher retained net system productivities over time (Figure 2). At lower 
mutation frequencies the differences between the net system productivities of plants reproducing by the same means with difering ploidy declined or disappeared (Figure 3).

The effect of renroduction mode on net system productivity in nonflare conditions.

Various plant species can reproduce by a number of modes of reproduction, both natural and man controlled. The node of reproduction selected for a plant could affect its net system productivity over time. The effect of varying reproduction modes at a constant mutation frequency were pronounced in this study. High ploidy plant systems utilizing modes of reproduction that select out recessive and leaky mutant genes with higher efficiencies (vegetative and cross fertilization) retained highest net system productivity (Figure 4). Lower ploidy plant systems in vegetatively reproducing plants retained higher net system productivities than cross fertilizing systems and self fertilizing systens retained ligher net system productivities than anther cultures (Figure 5). Figure 5 also demonstrates the effect of limited closed systems and randomness on the effect of mutation on net system productivity. In general, cross fertilizing plants retained higher net system productivities than self fertilizing plants. Ilowever in this specific case (Figure 5) a random event skewed the net system productivity of one or the other of the plants utilizing these reproduction modes. This has important implications for predicting events in a CELSS. Limited closed systems are susceptible to random events and events like this should be accounted for when planning system production capacity.

\section{The effect of viability thresholds on net system productivity in nonflare conditions.}

The viability threshold of a plant aftects the ability of a RU to germinate. At viability levels below the viability threshold, a RU will not germinate. This is a reflection of system complexity and gene redundancy. More complex systems have higher viability thresholds due to the higher probability of mutations disrupting the germination process. Systems with higher gene redundancies have lower viability thresholds. Mutations affecting RU germination tend to be selected out of the system as they manifest themselves. If a $\mathrm{RU}$ does nol germinate, another $\mathrm{RU}$ can be selected to take its place because of the short time frame involved. Hence the number of plants initially found in the system is not generally affected by low or medium levels of RU viability. Losses in net productivity occur by the loss of RUs which failed to germinate, unless the RU is not a food product. Such was the case in this study with plants reproducing by anther culture. In all other plant systems, net system productivity declined with higher viability thresholds (Figure 6). These systems represent those with more complex germination processes. This loss of net productivity was due to RUs that failed to germinate (Figure 7 ). The effect was less pronounced at higher ploidy (and hence higher levels of gene redundancy) with fewer RUs failing to germinate for the reproduction modes affected (Figure 8).

\section{The effect of lethality thresholds on net system productivity in nonflare conditions.}

Lethality thresholds affect the ability of a germinated RU to reach productive status. At lethality levels below the lethality threshold, a germinated RU will not reach productive status. In a fashion analogous to viability thresholds, this is a refection of system complexity and gene redundancy. Unlike viability threshold effects, the effects of lethality thresholds appear after the total plant number has been fixed. These effects are not correctable by simply regrowing another plant to replace the expired plant, as the affects occur well into the process of growth. Plants with higher lethality thresholds for all reproductive modes had lower net system productivities over time as compared to those with lower thresholds (Figure 9). IIigher ploidies demonstrated less net difference in net systenı productivities between plants with different lethality thresholds.

\section{The Eftect Or Solar Flares}

During periods of increased solar activity, the radiation fuxes encountered by a CELSS will increase the mutation rate of plants found in a CELSS for short periods of time. While space vehicle inliabitants will most likely have some place of refuge that is adequately shiclded, the size of CELSS units will make shielding unlikely due to mass restrictions.

\section{The eflect of fares without selectivity.}

Solar flares occur infrequently and with large variations in magnitude. They are characterized by short term increases in radiation that then subside back to nonflare levels after a period of time. In these simulations, flares were assumed to affect one generation only. Examination of situations where flares occur revealed that for all reproductive modes at any ploidy, fares resulted in immediate short term reductions in net system productivity followed to different degrees by some level of recovery. The productivity of plant syslems after recovery from flares usually eventually equaled the net system productivity of plant systems operating in conditions where fares did not occur (Figure 10). However the sliort term loss of net system productivity due to flares would in most cases cause a system failure wilh its associated results.

\section{The eflect of ploidy on uet system productivity after flares.}

As described above all systems experience net system productivity losses in the generations inmediately following a flarc. Plant systems with higher ploidy suffered smaller initial declines in net system productivity, and recovered more rapidly and generally to a higher net system productivity than plants with lower ploidy (Figure 11). This recovery is not surprising since the chances of producing simultaneous mutations at the same loci should be lower in plants with higher ploidies. 
The effect of reurorluction mode on net system system productivity in a flare situation.

Similar trends in net system productivity when varying reproduction mode were noted as without flares (Figure 12). Flares however caused large net system productivity losses immediately after the flare. These initial net system productivity losses were not found in nonflare conditions to the same extent.

\section{Selectivity EfTect On Retention Of Net Productivity}

Sclective breeding practices can be employed in closed systems to select for reproduction only those plants that have some hight predetermined level of productivity. In all the cases described above no selection was employed in determining which plants wcre allowed to reproduce. Systems in which some level of selectivity is introduced must have some form of highly automated selection procedure introduced in addition to the automation probably required to operate the CELSS. For this extra automation to be justified clear benefits of selective breeding must be realized.

The effect of selectivity on net system productivity in systems emploving various reproduction modes in constant radiation (nonflare) conditions.

Selecting during reproduction only those plants with known high productivity and excluding those with measurable poor productivity may be a way of combating net system productivity declines due to the effects of radiation. Whether selection occurs or not and the degree to which selection is employed will both affect the net result. In general all plants of any ploidy reproducing asexually by vegetative or anther culture means retained higher levels of net system productivity by employing any level of selectivity. The retention of net system productivity in these cases varied directly with the degree of selectivity employed (Figure 13). Plants of any ploidy reproducing by sexual means (self or cross fertilization) only had net system productivity enhanced by high degrees of selectivity. Lower levels of selectivity had no advantage over nonselective reproduction (Figure 14). Note that in all cases selection of breeding stock had no effect on system productivity when very high mutation rates prevailed throughout the lifetime of the system (Figure 15).

Flares and selectivity.

By being highly selective between generations, the recovery from flares was enhanced greatly over nonselected or partially selected plant systems (Figure 16).

\section{CONCLUSION}

The effect of the space environment on the multi-generational propagation of plants is unknown. However, with respect to the effect of radiation on net system productivity of a plant based CELSS, the selection of plants with ploidies, reproduction modes, and genotypes that minimize potential effects would be advised. To produce a more accurate prediction of the performance of a specific plant in a space based CELSS, more delailed computer models are recommended. A model of a specific plant species should be supported with actual data from a limited multi-generation experiment of the plant being exposed to radiation conditions similar to those found in space.

\section{ACKNOWLEDGMENTS}

The authors would like to thank T. Chen, M. Lazar, P. Nagarajan, and D. Ward for helpful comments regarding the manuscript.

\section{REFERENCES}

1. R.D. MacElroy, N.V. Mactello, and D.T. Smernoff (Editors). NASA TM 88215 (1986)

2. A.J. Merkys, R.S. Lawinavičius, and D.V. Švegżdiene, Adv. Space Res. 4, \#10, 55 (1984)

3. I.D. Anikeeva, L.N. Kostina, and E.N. Vaulina, Adv. Space Res. 3, \#8, 129 (1983)

4. Y. Gaubin, H. Planel, G. Gasset, B. Pianezzi, J. Clegg, E.E. Kovalev, L.V. Nevgodina, E.N. Maximova, A.T. Miller, and M. Delpoux, Adv. Space Res. 3, \#8, 135 (1983)

5. L. Kostina, I. Anikeeva, and E. Vaulina, Adv. Space Res. 4, \#10,65 (1984)

6. J. Bayonove, M. Burg, M. Delpoux, and A. Mir, Adv. Space Res. 4, \#10, 97 (1984)

7. E. Vaulina, I. Anikceva, and L. Kostina, Adv. Space Res. 4, \#10, 103 (1984) 
Figure I Net productivity versus plant generation for plans reprevlucing al differem mutuation frequencies. Self fertilization reprowluction noods. Diploid plants. Symbols and abbreviations: TRIANGLE = mustion frequency of 0.I: STAR = muation frequency of (O.): SQUARE = mulation frequency of (0.001: and DIAMOND = mutation frequemy of 000x).

Figure 2 - Net productivity versus plant generation for plants of differing ploidy. Mutation frequency $=0.1$. Vegetative $r e$ production mode. Symbols and abbreviations: TRIANGLE diploid: STAR = tetraploid; and SQUARE = hexaploid.

Figure 3 - Net productivity versus plant generation for plants of differing ploidy. Mutation frequency $=0.01$. Self fertiliztion reproductive moile. Symbols and abbreviations as in Figure 2.

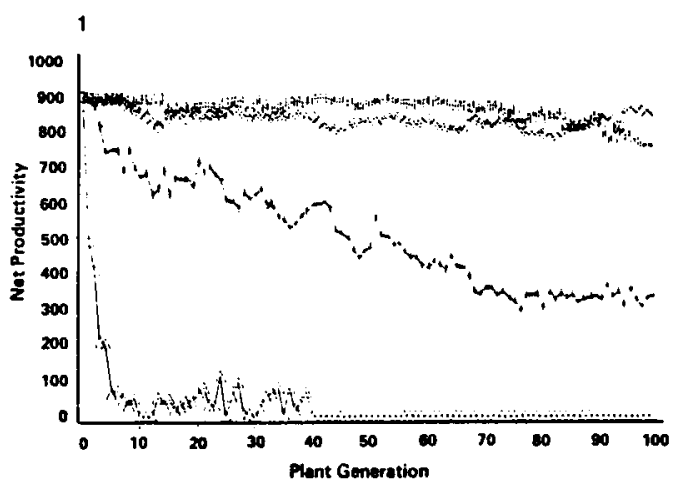

2

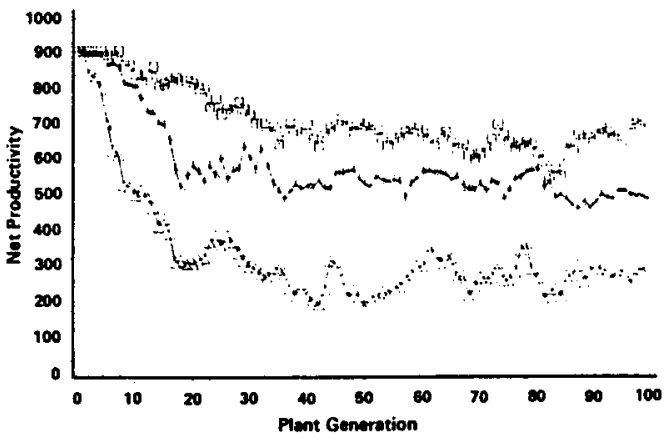

3
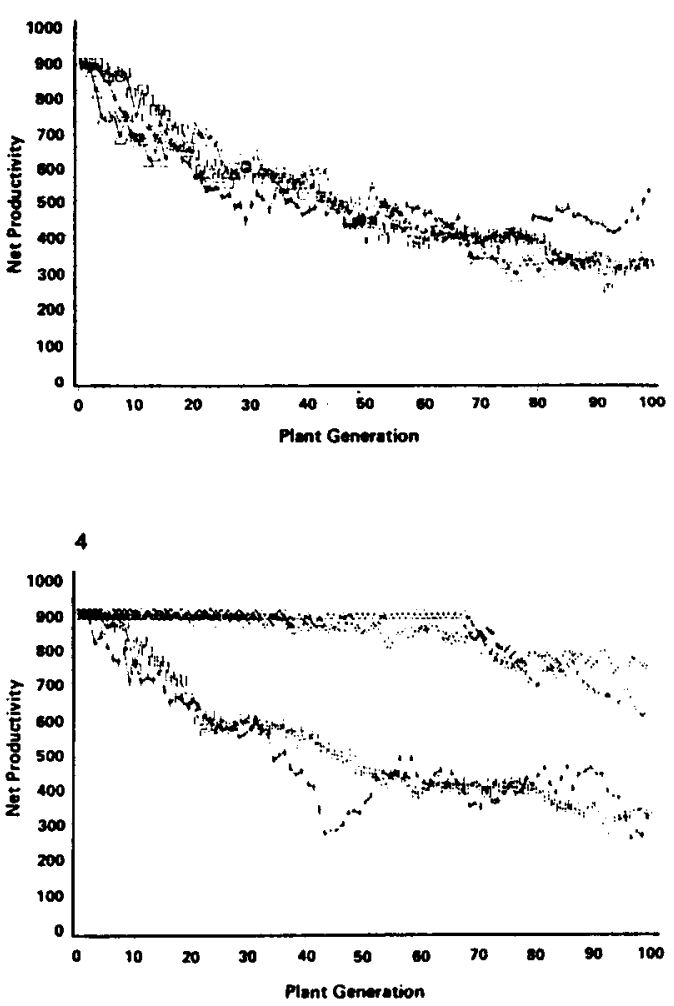
Figure 5 - Net groductivily versus plant generation fort plants using various undes of reproduction. Mutation frequency = 0.01. Tetrapleid plants, Synubols and abbreviations as in Figure 4.

Figure 6 - Net productivity versus plant generation for plants with differing viability thresholds. Mutation frequency $=0.1$. Cross fertilization reproductive mode. Diploid plants. Symbols and abbreviations: TRIANGLE = viability threshold of 0.2 : STAR $=$ viability threshold of 0.4; SQUARE $=$ viability threshold of 0.6; and DIAMOND = viability threshold of 0.8 .

Figure 7 - RUs failing to germinate versus plant generation for plants with differing viability thresholds. Mutation frequency - 0.1. Cross ferilization reproductive mode. Diploid plants. Symbols and abbreviations as in Figure 6.

Figure 8 - RUs failing to germinate versus plant generation for plants with differing ploidy. Mutation frequency $=0.1$. Cross fertilization reproductive mode. Viahility threshold $\mathbf{= 0 . 4}$. Symbols and abbreviations as in Figure 2.
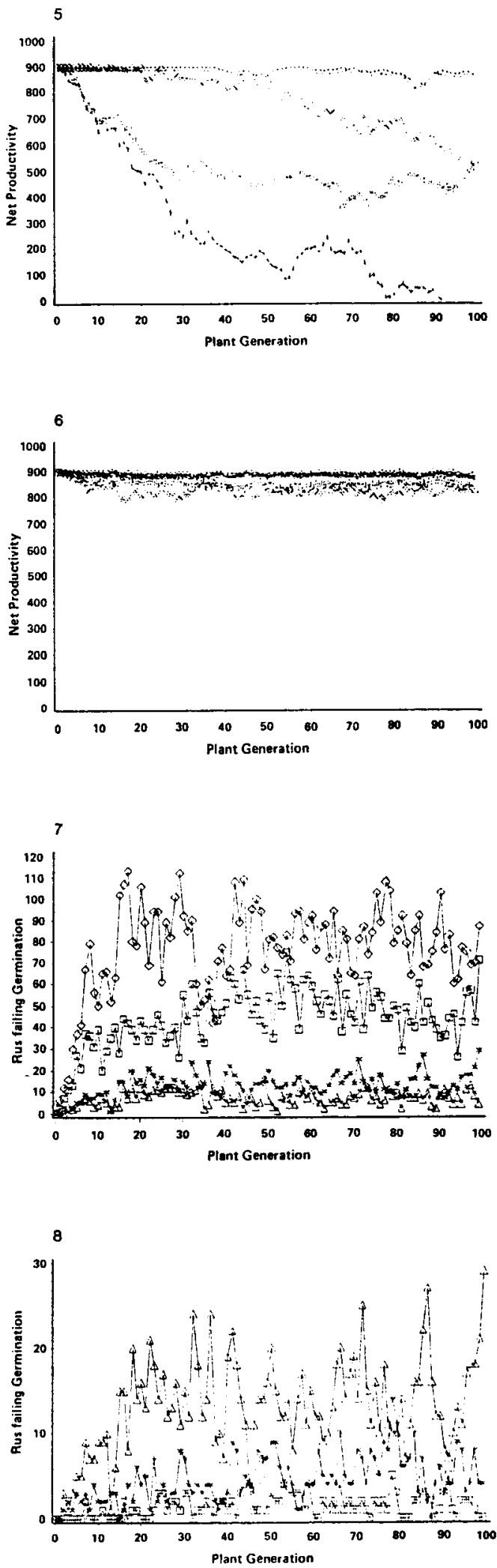
Figure 9. Net productivity versus plant generation firr plants with different lethality threstustds. Mutation frequency $=0.1$. Dipkoid plants. Cross fertilization reproduction mode. Symbols and abtreviations: TRIANGLE $=$ Icthality threstold of 0.2 . STAR = lech:dity threshoidd of (0.4: SQUARE = lethulity threshold of 0.6: anI DIAMOND = lethality threshold of 0.8 .

Figure 10 - Net syseem productiviry versus plant genention for plants under conditions with or without solar flares. Cross fertilization reproduction mode. Diploid plants. Mutation frequency for plants under conditions of a solar flare for first generation $=0.5$, for generation 2-100 $=0.01$. Mutation frequency for plants without solar flares $=0.1$. Symbols and abbreviations: STAR = without flare; and TRIANGLE = with flare.

Figure 11 - Net productivily versus plant generation for plants with differing ploidy under conditions of solar flares in the first generation. Mutation frequency in generation $1=0.5$. mutation frequency for generations $2-100=0.01$. Self ferilization reproductive mode. Symbols and abbreviations as in Figure 2.

Figune 12 - Net productivity versus plant generation for plants uxing various modes of reproduction under conditions of solar fares in the first generation. Mutation frequency in generation $1=0.5$. nutation frequency for generations 2-100 $=0.01$. Hexaploid plants. Symbols and abbreviations as in Figure 4.
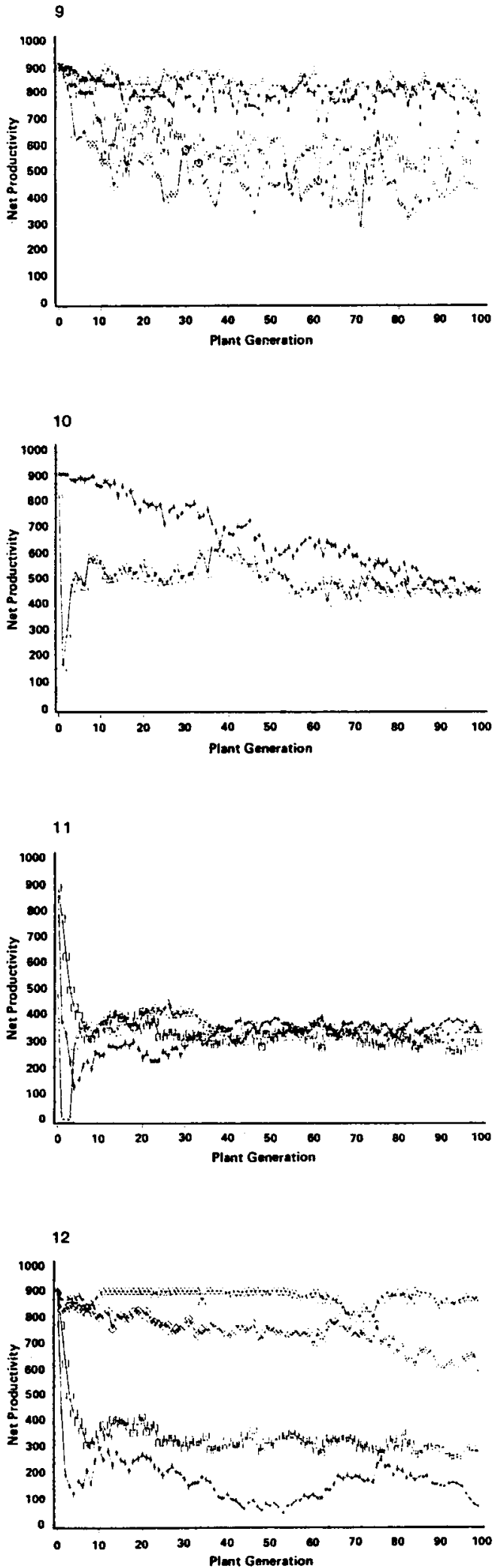
Figure 13 - Net preduclivity versus plant generation for plants meproducing with different levels of system selectivity during reproduction. Veyetative moxk of reprexluction. Diploid plants. Mutation frequency $=0.01$. Symbols and abbreviatioms: TRIANGLE = selectivity level of 0; SQUARE = selectivity level of 5; STAP = selectivity level of 10

Figure 4 - Net productivity versus plant generntion for plants reproducing with different levels of system selectivity during reproduction. Self fertilization mode of repmeduction. Diploid plants. Mutation frequency $=0.01$. Symbols and abbreviations as in Figure 13.
13
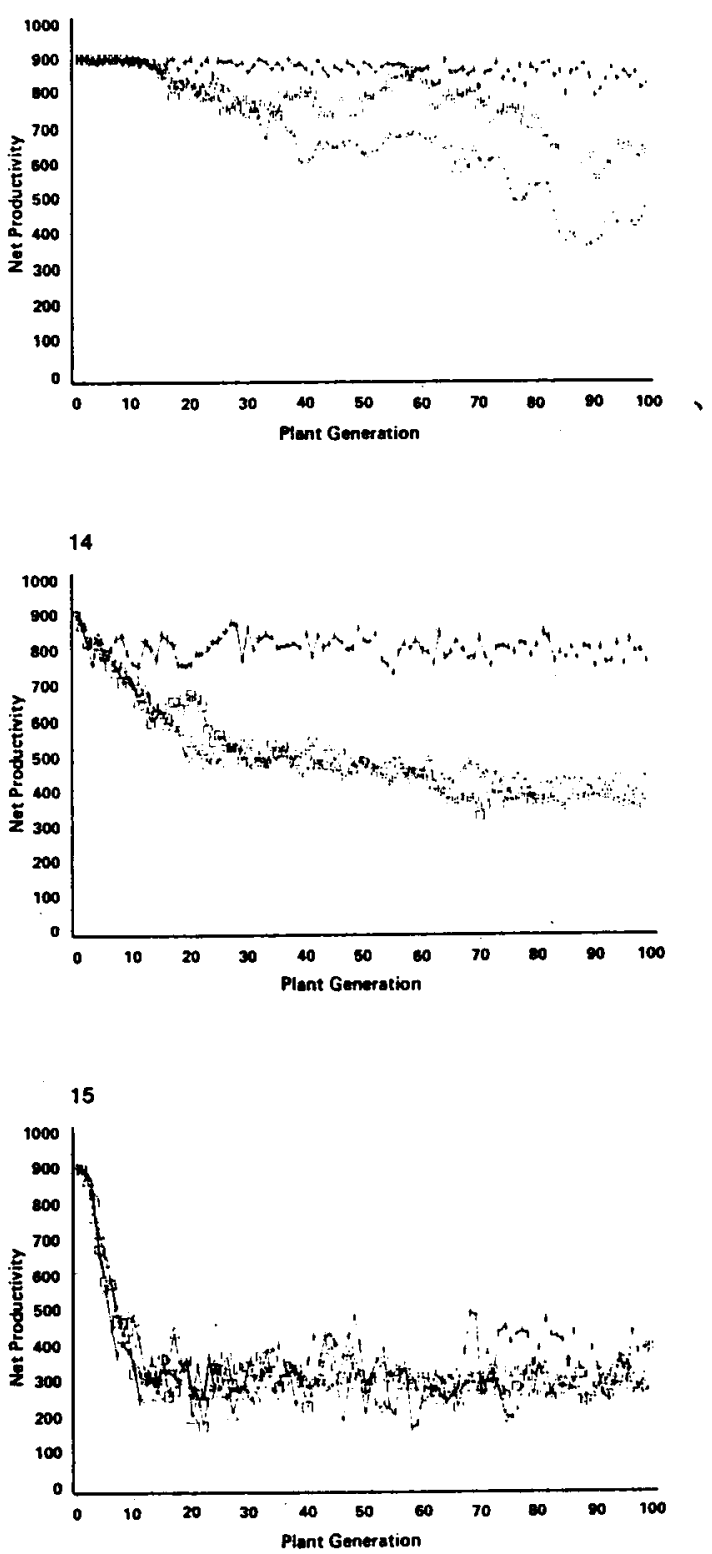

16

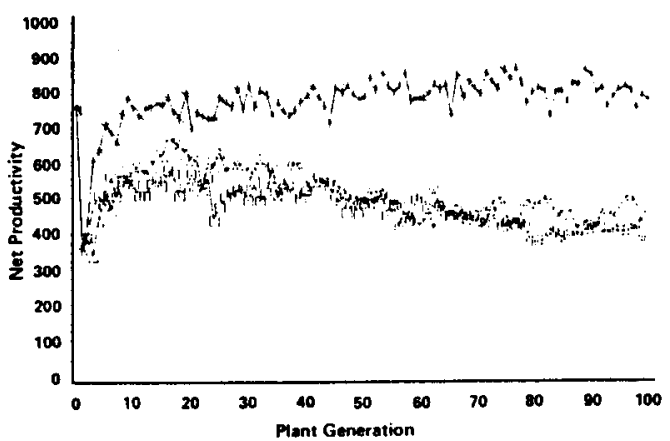

